Rapid Commun. Mass Spectrom. 2013, 27, 476-480

(wileyonlinelibrary.com) DOI: 10.1002/rcm.6471

\title{
Surface-activated chemical ionization-electrospray mass spectrometry in the analysis of urinary thiodiglycolic acid
}

\author{
Giovanni Puccio ${ }^{1}$, Paolo Brambilla ${ }^{2}$, Matteo Conti ${ }^{3}$, Desirée Bartolini ${ }^{4}$, Douglas Noonan ${ }^{5,6}$ and \\ Adriana Albini ${ }^{6 *}$ \\ ${ }^{1}$ Emmanuele Scientific Research Association, Palermo, Italy \\ ${ }^{2}$ Hospital of Desio, Milan, Italy \\ ${ }^{3}$ Sant'orsola-Malpighi Hospital, Bologna, Italy \\ ${ }^{4}$ IRCCS Arcispedale S. Maria Nuova, Reggio Emilia, Italy \\ ${ }^{5}$ University of Insubria, Varese, Italy \\ ${ }^{6}$ IRCCS MultiMedica, Polo Scientifico e Tecnologico, Milan, Italy
}

RATIONALE: Thiodiglycolic acid (TDGA) is a urinary metabolite of the oxazaphosphorine class of chemotherapeutics, in particular of ifosfamide. Ifosfamide metabolism generates chloroacetaldehyde (CAA), a toxic compound associated with neurotoxicity, nephrotoxicity, urotoxicity and cardiotoxicity. CAA, in turn, interacts with cellular thiol groups leading to GSH depletion, cell death and generation of thiodiglycolic acid (TDGA), as a final product. TDGA is mainly excreted in the urine. The ability to accurately measure TDGA in urine, therefore, will be a useful way of monitoring exposure to ifosfamide during chemotherapy.

METHODS: TDGA in urine samples was measured with liquid chromatography coupled to mass spectrometry (LC/MS) by means of a novel Surface-Activated Chemical Ionization-Electrospray (SACI-ESI) or a classical ESI ion source alone. RESULTS: The SACI-ESI and ESI alone based methods for analysis of urinary TDGA were optimized and compared. A strong reduction in matrix effect together with enhanced quantification performances was obtained with the SACI-ESI when compared with ESI. In particular, an increase in quantification precision (from 85 to 95\%) and accuracy (from 59 to $90 \%$ ) were observed, which allowed for optimal detection of TDGA.

CONCLUSIONS: The LC/SACI-ESI-MS approach provides a very sensitive and quantitative method for the analysis of TDGA. Thanks to the enhancement in sensitivity and matrix effect reduction, the SACI-ESI source enables the use of a relatively low-cost ion-trap mass spectrometer in the analysis of this toxicity biomarker in urine. Due to these characteristics, this approach would constitute an invaluable tool in the clinical laboratory, for measuring TDGA and other toxicity related biomarkers of chemotherapy with proper sensitivity and accuracy. Copyright @ 2012 John Wiley \& Sons, Ltd.

The oxazaphosphorines (cyclophosphamide, ifosfamide and trofosfamide) are widely used in clinical practice for their anti-tumor and immuno-modulatory activities. These drugs, however, have associated neurotoxicity, nephrotoxicity, urotoxicity and cardiotoxicity. ${ }^{[1-7]}$ Cytochrome P450 metabolism of ifosfamide leads to formation of highly reactive metabolites, in particular acrolein and chloroacetaldehyde, which are considered to be responsible for the major associated toxicities. $^{[2,4,5,7]}$ The interaction of chloroacetaldehyde with cellular molecules containing thiol groups (e.g. glutathione and cysteine) results in the generation of the downstream metabolite thiodiglycolic acid (TDGA) ${ }^{[6]}$ which is also related to toxicity. ${ }^{[5,7]}$ The cascade of metabolic events that leads to CAA and TDGA formation is given in Scheme 1. TDGA is a low molecular weight metabolite (MW: $150 \mathrm{Da}$ ) with two highly polar acid groups (see Scheme 1). CAA is very difficult to

* Correspondence to: A. Albini, IRCCS MultiMedica, Polo Scientifico e Tecnologico, Via Fantoli 16/15, 20138 Milan, Italy. E-mail: albini.adriana@gmail.com quantify due to high reactivity with thiol groups that leads to its rapid depletion. ${ }^{[1]}$ TDGA is a quantitative reaction product of CAA, detectable and quantifiable in urine due to a better stability and higher polarity. It represents a useful biomarker of CAA elimination. Since the metabolism of ifosfamide to CAA depends on numerous inducible cytochrome P450 isoforms, measurement of TDGA in the urine provides a simple approach to estimate CAA generation during ifosfamide chemotherapy, allowing for adjustments in dosage and schedule. Different methods have been developed to quantify TDGA in serum and urine. ${ }^{[8-12]}$ One valid approach was to quantify TDGA using capillary electrophoresis coupled with an ultraviolet absorbance detector (CE-UV). This method exhibits high sensitivity (the limit of quantification (LOQ) between 5-10 micrograms/L) ${ }^{[12]}$ but with lower selectivity as compared to a separation technology coupled to a mass spectrometric analyzer. Other groups developed approaches based on gas chromatography/mass spectrometry (GC/MS). ${ }^{[8,10,11]}$ However, despite its high selectivity and sensitivity, time-consuming sample extraction and preparation procedures make it unsuitable for modern high 

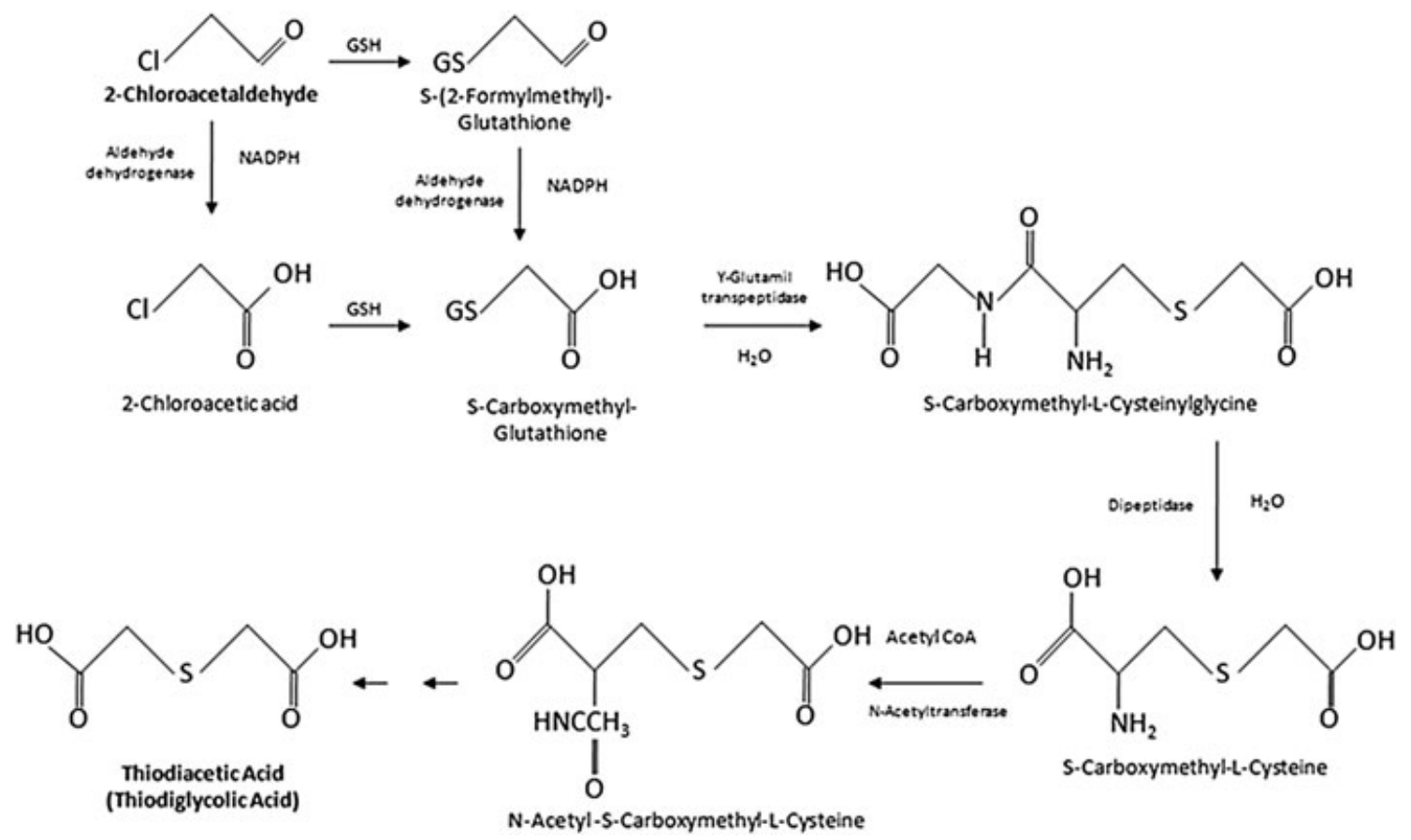

Scheme 1. Cascade of metabolic events that leads to CAA and TDGA formation.

productivity clinical laboratories. Recently, two different approaches based on ultra-performance liquid chromatography/high-resolution and mass accurate time-of-flight mass spectrometry (UPLC/TOF-MS) technology have been developed to quantify different chemotherapy-related toxicity biomarkers, including TDGA. ${ }^{[9,13]}$ This technology makes it possible to obtain a high chromatographic resolution, reducing the matrix effect ionization effect ${ }^{[14]}$ that lowers quantification precision and accuracy in the analysis of biological samples. The high mass accuracy of the TOF analyzer makes it possible to obtain high selectivity and sensitivity. Despite the remarkable performance of this solution, its costs are quite high for many laboratories' budget. Therefore, development of less expensive, although performing technology would be an interesting option for monitoring toxic metabolites.

One approach that has been explored to efficiently reduce the matrix effect without using an UPLC system is to employ low voltage ionization sources such as the no discharge atmospheric pressure chemical ionization (ND-APCI), ${ }^{[15,16]}$ a detection technology developed by Cristoni et al. ${ }^{[17,18]}$ In this case, an APCI source was employed to ionize analytes without using high-voltage corona discharge. Under these conditions both the spectrum chemical noise and matrix effect significantly decrease, leading to a stable analyte quantification. An improvement of ND-APCI, now named surface-activated chemical ionization (SACI), has been developed in order to increase the signal/noise (S/N) ratio. ${ }^{[19]}$ In SACI the ionization efficiency is increased by inserting a surface placed at low voltage (50-400 V) that activates a gas-phase solvent polarization and proton reaction mechanism. Recently, the SACI phenomenon has been exploited to enhance the performance of electrospray ionization (ESI) further in terms of matrix effect reduction, quantification precision and accuracy and sensitivity (SACI-ESI). ${ }^{[20,21]}$ In the SACI-ESI ionization approach, sample ionization involves both the Ion Evaporation (IEM) model and Charge Reaction (CRM) models, ${ }^{[22]}$ along with the impact of a quota of charged droplets with the SACI surface. This improves the ion gasification efficiency. In addition, the electric field of the metallic surface in the SACI source, which is placed at an angle of about $45^{\circ}$ relative to the axis of the ESI spray cone, acts as an ion deflector, pushing ions towards the entry orifice of the mass analyzer. This allows a reduction in the chemical noise due to the extra-charged solvent environment and an increase in analyte ion signal due to the high efficiency of ESI ionization combined with the SACI ion impact evaporation and focalization effects.

Here we describe a new high-throughput approach (5 min of total sample run time) based on the highly efficient and less expensive liquid chromatography (LC)/SACI-ESI tandem mass spectrometry (MS/MS) method that was developed to quantify TDGA as a toxicology biomarker. The new approach (LC/SACI-ESI-MS/MS) was compared with the classical liquid chromatography/electrospray ionization tandem mass spectrometry (LC/ESI-MS/MS) method, and the benefits, mainly in terms of matrix effect reduction, quantification, precision, accuracy, sensitivity and sample throughput, are shown.

\section{EXPERIMENTAL}

\section{Chemicals}

The normal and deuterated (D3) standard TDGA as well as formic acid were purchased from Sigma-Aldrich (Milan, Italy). Methanol and water were purchased from Romil (Cambridge, UK). 
Urine sample preparation

Urine $(100 \mu \mathrm{L})$ was diluted 2-fold with doubly distilled water and two different urine samples that contained spiked $500 \mathrm{ng} / \mathrm{mL}$ TDGA were used during the development of the LC/MS and MS/MS procedures.

A calibration curve was prepared in water with 1, 2, 5, 20 and $50 \mathrm{ng} / \mathrm{mL}$ TDGA. The matrix effect was determined by the addition of TDGA standards at the concentrations of 5 and $50 \mathrm{ng} / \mathrm{mL}$. The level of TDGA in each sample was determined from the ratio of the peak area of TDGA to that of the TDGAD3 internal standard.

\section{Chromatography}

A fast isocratic chromatography was performed using an Ultimate 3000 HPLC system (DIONEX, Sunnyvale, CA, USA) with an Aquasil C18 LC column $(100 \times 2.1$ mm, $3 \mu \mathrm{m}, 100 \AA$; Thermo Scientific, San Jose, CA, USA).

During the analysis an isocratic condition was employed using $\mathrm{H}_{2} \mathrm{O}+0.5 \%$ (v/v) formic acid (phase A)/acetonitrile $\left(\mathrm{CH}_{3} \mathrm{CN}+0.5 \%(\mathrm{v} / \mathrm{v})(98: 2)\right.$ formic acid (phase B). The acquisition time was $3 \mathrm{~min}$. After five analyses, a column washing step ( $0 \mathrm{~min}, 2 \% \mathrm{~B} ; 0$ to $1 \mathrm{~min}$, a linear gradient of $0 \%$ to $80 \% \mathrm{~B}$; 1 to $5 \mathrm{~min}$, isocratically $80 \% \mathrm{~B}$ ) was used to clean the column from residual urine metabolites; followed by re-equilibration to starting conditions for a further $5 \mathrm{~min}$. Considering this washing step the average total run time (TRT) for each analysis was $5 \mathrm{~min}$. The flow rate was $100 \mu \mathrm{L} / \mathrm{min}$.

\section{Mass spectrometry}

Mass spectra were acquired using an LTQ ion trap spectrometer (Thermoelectron, San Jose, CA, USA) in negative acquisition mode. Three microscans for each spectrum were acquired in the positive mode. A rolling average of four spectra was also used. ESI and SACI-ESI ionization approaches were employed, which were coupled to MS and MS/MS analyzers. The ion isolation width used for MS/MS was $2 \mathrm{~m} / \mathrm{z}$ units. The collision energy was set to $60 \%$ of the maximum value. The ESI spray voltage was $4500 \mathrm{~V}$, the nitrogen drying gas temperature was $350{ }^{\circ} \mathrm{C}$ and its flow was $8 \mathrm{~L} / \mathrm{min}$. The nebulizing gas (nitrogen) flow was $10 \mathrm{~L} / \mathrm{min}$. The SACI-ESI spray voltage was $1500 \mathrm{~V}$, the nitrogen drying gas temperature was $350{ }^{\circ} \mathrm{C}$ and its flow was $8 \mathrm{~L} / \mathrm{min}$. The SACI surface voltage was $150 \mathrm{~V}$. The nebulizing gas (nitrogen) flow was $10 \mathrm{~L} / \mathrm{min}$.

\section{Data analysis}

Xcalibur software (Thermofisher, San Jose, CA, USA) was used for data acquisition. Qual- and QuanBrowser (ThermoFisher, San Jose, CA, USA) were employed for data elaboration. The signal/noise $(\mathrm{S} / \mathrm{N})$ ratio was calculated using the Root Mean Square (RMS) algorithm. ${ }^{[23]}$

\section{RESULTS AND DISCUSSION}

Initial experiments employed a direct infusion. A standard $500 \mathrm{ng} / \mathrm{mL}$ TDGA aqueous solution was analyzed using both the ESI and SACI-ESI approaches. Figures 1(a) and 1(b) show the direct infusion spectra. As can be seen, the $\mathrm{S} / \mathrm{N}$ ratio of

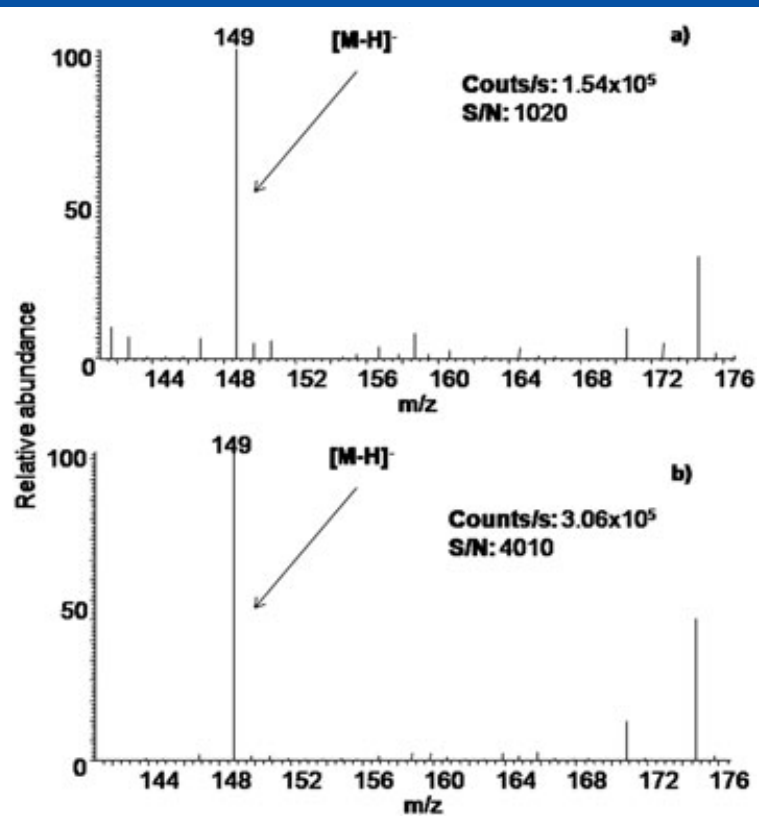

Figure 1. Direct infusion spectra of a $500 \mathrm{ng} / \mathrm{mL}$ aqueous solution of TDGA standard obtained using both the (a) ESI and (b) SACI-ESI approaches. The syringe sample flow rate was $10 \mu \mathrm{L} / \mathrm{min}$.

the $[\mathrm{M}-\mathrm{H}]^{-}$ion signal at $m / z 149$ is definitely higher using SACI-ESI. Under ESI conditions, the RMS calculated S/N ratio was 1020 while with SACI-ESI the value increased to 4010. It must be emphasized that the increase in $\mathrm{S} / \mathrm{N}$ ratio is mainly due to two joined contributions:

A) The higher $[\mathrm{M}-\mathrm{H}]^{-}$signal intensity that increases from $1.54 \times 10^{5}$ under ESI conditions to $3.06 \times 10^{5}$ using SACI-ESI; this phenomenon is due to the sum of electrospray and SACI solvent polarization ionization effects.

B) The lower spectrum chemical noise observed under SACI-ESI conditions. Under the ESI conditions an average background noise of $1.2 \times 10^{2}$ was observed while in SACI-ESI it drops down to $5.2 \times 10^{1}$. This decrease is mainly due to the lower amount of solvent cluster ion species usually obtained under the lower voltage SACI-ESI ionization conditions. ${ }^{[18,19,21]}$

The MS/MS spectrum was obtained by fragmentation of the $[\mathrm{M}-\mathrm{H}]^{-}$precursor ion of TDGA at $m / z 149$ in order to increase the $\mathrm{S} / \mathrm{N}$ ratio and instrumental specificity. This fragmentation spectrum is shown in Fig. 2. An abundant $\mathrm{CO}_{2}$ neutral loss was observed, leading to the fragment ion at $m / z 105$.

A high-throughput 3-min isocratic chromatography was performed under both LC/ESI-MS/MS and LC/SACIESI-MS/MS conditions in order to analyze urine samples and evaluate the matrix effect suppression. Isocratic conditions were maintained using an $\mathrm{H}_{2} \mathrm{O} / \mathrm{CH}_{3} \mathrm{CN}$ elution phase with $0.5 \%(\mathrm{v} / \mathrm{v})$ formic acid. This formic acid concentration is fundamental. On one hand, the acid solution environment should help the deprotonation of the TDGA acid molecule in the ionization source while, on the other hand, it makes it possible to better retain compounds on the $\mathrm{C} 18$ stationary phase of choice. During the chromatographic separation, the TDGA acid groups 


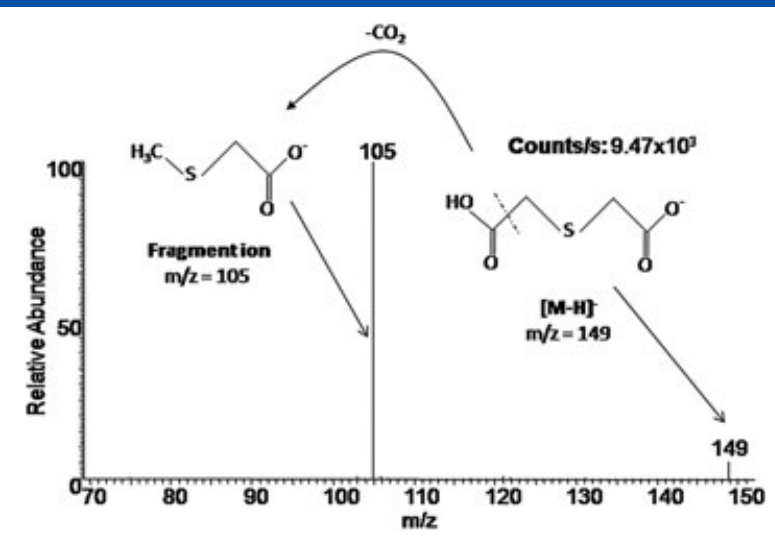

Figure 2. Direct infusion MS/MS spectrum of the $[\mathrm{M}-\mathrm{H}]^{-}$ precursor ion of TDGA at $m / z 149$. The syringe sample flow rate was $10 \mu \mathrm{L} / \mathrm{min}$.

(see Scheme 1) are protonated, decreasing the analyte polarity and consequently producing improved column retention. The SACI polarization effect taking place in the SACI-ESI ionization source helps to reduce the TDGA proton affinity, consequently increasing ionization efficiency even under acid solvent conditions. Figures 3(a) and 3(b) show the LC/ESI-MS/MS ion extraction mass chromatogram obtained by monitoring a final concentration $50 \mathrm{ng} / \mathrm{mL}$ of TDGA in spiked bi-distilled water and urine, in both cases diluted 1:10 with bi-distilled water before analysis. In this case, a strong matrix suppression effect is observed in urine. In fact, the chromatographic RMS $\mathrm{S} / \mathrm{N}$ ratio undergoes about a 3-fold decrease, the measured $\mathrm{S} / \mathrm{N}$ ratio dropping from a value of 300 in water to a value of 90 in urine.

Figures 3(c) and 3(d) show the same analyses performed with the LC/SACI-ESI-MS/MS apparatus. Not only is the $\mathrm{S} / \mathrm{N}$ ratio obtained for the compound in water about two times higher than that obtained with the optimized LC/ESIMS/MS conditions, but a clear reduction in matrix ion
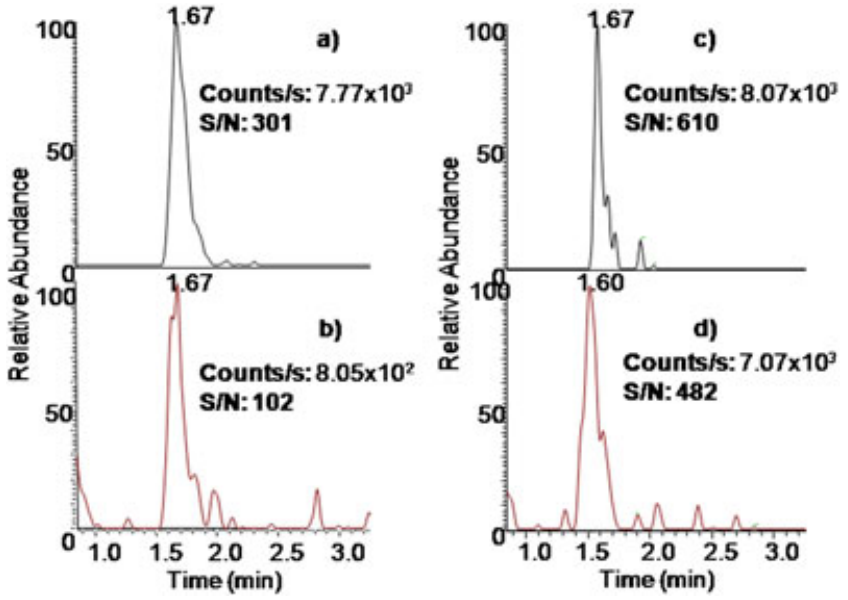

Figure 3. LC/ESI-MS/MS ion extraction mass chromatograms obtained by monitoring a final concentration $50 \mathrm{ng} / \mathrm{mL}$ of TDGA in (a) bi-distilled water and (b) spiked urine diluted 1:10 with bi-distilled water. LC/SACI-ESI-MS/MS ion extraction mass chromatograms obtained by monitoring a final concentration $50 \mathrm{ng} / \mathrm{mL}$ of TDGA in (c) bi-distilled water and (d) spiked urine diluted 1:10 with bi-distilled water. The sample injection volume was $10 \mu \mathrm{L}$. suppression effect is also observed. In fact, the reduction in the $\mathrm{S} / \mathrm{N}$ ratio in urine is only of $21 \%$, the measured $\mathrm{S} / \mathrm{N}$ ratio dropping from a value of 610 in water to a value of 482 in urine. This was probably due to the fact that the SACI-ESI source can be operated at a lower voltage than that used with the ESI source alone. It must be emphasized that a weak thyodyglicolic acid retention time shift of 0.07 min has been observed when comparing the bi-distilled water and urine analyses (Figs. 3(c) and 3(d)). This is probably due to progressive column stationary phase degradation due to matrixcolumn interactions. In particular, the column life under the experimental conditions used here was approximately 550 urine samples.

We then compared the LC/ESI- and LC/SACI-ESI-MS/MS ionization approaches for quantification performance in terms of limit of detection (LOD), limit of quantification (LOQ), linearity range, precision and accuracy. The results obtained are shown in Table 1. The SACI-ESI approach exhibits, in general, better performance with respect to the ESI approach alone. In particular, a strong increase in sensitivity was observed: the absolute LOD value changed from $5 \mathrm{ng} / \mathrm{mL}$ to $1 \mathrm{ng} / \mathrm{mL}$, while the absolute LOQ value went from $10 \mathrm{ng} / \mathrm{mL}$ to $3 \mathrm{ng} / \mathrm{mL}$, with ESI and SACI-ESI, respectively. Another strong benefit was obtained in terms of quantification precision and accuracy. These benefits are likely due to the reduction in the matrix effect achieved under the SACI-ESI operating conditions. All these parameters were measured by analyzing two urine samples spiked with different concentrations of TDGA (50 and $200 \mathrm{ng} / \mathrm{mL}$ ). Table 2 shows the results obtained in terms of precision and accuracy for each sample using both the LC/ESI- and LC/SACI-ESIMS/MS approaches. The average precision increased from 85 to $95 \%$ and average accuracy from 59 to $90 \%$ with LC/ SACI-ESI-MS/MS.

\section{CONCLUSIONS}

A rapid, optimized and cost-effective method for the analysis of TDGA, a biomarker of ifosfamide toxicity, in urine has been developed. The new method is based on the innovative SACI-ESI ionization system coupling LC with a relatively inexpensive ion-trap MS analyzer. This novel technology leads to a significant increase in performances, both in terms of sensitivity (LOQ) and quantification (LOD, precision and accuracy), when compared with a classical ESI ionization system.

Future studies will be devoted to testing this approach in monitoring oxazaphosphorine metabolites during chemotherapy.

Table 1. Comparative table reporting the limit of detection (LOD), limit of quantification (LOQ), linearity range, precision and accuracy of the LC/ESI- and LC/SACI-ESIMS/MS approaches

\begin{tabular}{|lccc|} 
& $\begin{array}{c}\text { LOD } \\
(\mathrm{ng} / \mathrm{mL})\end{array}$ & $\begin{array}{c}\text { LOQ } \\
(\mathrm{ng} / \mathrm{mL})\end{array}$ & $\begin{array}{c}\text { Linearity range } \\
(\mathrm{ng} / \mathrm{mL})\end{array}$ \\
\hline ESI & 5 & 10 & $10-250$ \\
SACI-ESI & 1 & 3 & $3-250$ \\
\hline
\end{tabular}


Table 2. Quantification results achieved analyzing 15 urine samples spiked with different concentrations of TDGA, using both the LC/ESI- and LC/SACI-ESI-MS/MS approaches. The theoretical quantification value together with quantification accuracy and precision are also reported

\begin{tabular}{|c|c|c|c|c|}
\hline \multirow[b]{2}{*}{ Sample } & \multicolumn{2}{|c|}{ LC/ESI-MS/MS } & \multicolumn{2}{|c|}{ LC/SACI-ESI-MS/MS } \\
\hline & $\begin{array}{c}\text { Precision } \\
(\%)\end{array}$ & $\begin{array}{c}\text { Accuracy } \\
(\%)\end{array}$ & $\begin{array}{c}\text { Precision } \\
(\%)\end{array}$ & $\begin{array}{c}\text { Accuracy } \\
(\%)\end{array}$ \\
\hline Urine $A+50 \mathrm{ppb}$ & 90 & 49 & 93 & 88 \\
\hline Urine $A+200 \mathrm{ppb}$ & 80 & 50 & 99 & 92 \\
\hline Urine $B+50 \mathrm{ppb}$ & 92 & 70 & 91 & 85 \\
\hline Urine $\mathrm{B}+200 \mathrm{ppb}$ & 78 & 68 & 97 & 95 \\
\hline Average & 85 & 59 & 95 & 90 \\
\hline
\end{tabular}

\section{Acknowledgements}

The authors thank Dr. Giuseppe Galasso of Radical service laboratory for his advice and support. We are grateful to Paola Corradino for bibliographic searches and data management. These studies were supported by the Associazione Italiana per la Ricerca sul Cancro (AIRC) and Ministero Salute-Ricerca Finalizzata 2010 (to AA with Paola Erba), the MIUR Grande Progetto Strategico GPS DM28938, and MultiMedica Onlus (AA and DN).

\section{REFERENCES}

[1] A. Benesic, G. Schwerdt, R. Freudinger, S. Mildenberger, F. Groezinger, B. Wollny, A. Kirchhoff, M. Gekle. Kidney Blood Press. Res. 2006, 29, 280.

[2] N. Chen, K. Aleksa, C. Woodland, M. Rieder, G. Koren. Transl. Res. 2007, 150, 51.

[3] Y. T. Chen, C. I. Hsu, D. Z. Hung, I. Matsuura, J. W. Liao. Food Chem. Toxicol. 2011, 49, 1063.

[4] B. Giraud, G. Hebert, A. Deroussent, G. J. Veal, G. Vassal, A. Paci. Expert. Opin. Drug Metab. Toxicol. 2010, 6, 919.

[5] L. Hanly, N. Chen, M. Rieder, G. Koren. Expert Opin. Drug Saf. 2009, 8, 155.

[6] G. Schwerdt, A. Kirchhoff, R. Freudinger, B. Wollny, A. Benesic, M. Gekle. Pediatr. Nephrol. 2007, 22, 798.

[7] J. Zhang, Q. Tian, S. Yung Chan, S. Chuen Li, S. Zhou, W. Duan, Y. Z. Zhu. Drug Metab. Rev. 2005, 37, 611.

[8] U. Hofmann, M. Eichelbaum, S. Seefried, C. O. Meese. Drug Metab. Dispos. 1991, 19, 222.
[9] F. Li, A. D. Patterson, C. C. Hofer, K. W. Krausz, F. J. Gonzalez, J. R. Idle. Biochem. Pharmacol. 2010, 80, 1063.

[10] G. Muller, K. Norpoth, R. Eckard. Cancer Lett. 1979, 7, 299.

[11] G. Muller, K. Norpoth, R.H. Wickramasinghe. Int. Arch. Occup. Environ. Health 1979, 44, 185.

[12] E. Samcova, V. Kvasnicova, J. Urban, I. Jelinek, P. Coufal. J. Chromatogr. A 1999, 847, 135.

[13] F. Li, A. D. Patterson, C. C. Hofer, K. W. Krausz, F. J. Gonzalez, J. R. Idle. Biochem. Pharmacol. 2011, 81, 1043.

[14] C. Cote, A. Bergeron, J. N. Mess, M. Furtado, F. Garofolo. Bioanalysis 2009, 1, 1243.

[15] S. B. Turnipseed, W. C. Andersen, C. M. Karbiwnyk, J. E. Roybal, K. E. Miller. Rapid Commun. Mass Spectrom. 2006, 20, 1231.

[16] S. B. Turnipseed, W. C. Andersen, J. E. Roybal. J. AOAC Int. 2005, 88, 1312.

[17] S. Cristoni, L. R. Bernardi, I. Biunno, F. Guidugli. Rapid Commun. Mass Spectrom. 2002, 16, 1686.

[18] S. Cristoni, L. R. Bernardi, I. Biunno, F. Guidugli. Rapid Commun. Mass Spectrom. 2002, 16, 1153.

[19] S. Cristoni, S. Rubini, L. R. Bernardi. Mass Spectrom. Rev. 2007, 26, 645 .

[20] M. Conti, V. Tazzari, M. Bertona, M. Brambilla, P. Brambilla. Rapid Commun. Mass Spectrom. 2011, 25, 1552.

[21] I. Sogno, M. Conti, P. Consonni, D. M. Noonan, A. Albini. Rapid Commun. Mass Spectrom. 2012, 26, 1213.

[22] P. Kebarle. A brief overview of the present status of the mechanisms involved in electrospray mass spectrometry. J. Mass Spectrom. 2000, 35, 804.

[23] D. Green, J. Swets. Signal Detection Theory and Psychophysics. Peninsula Publishing, Los Altos, CA, 1988. 